\title{
Astrocyte elevated gene-1 regulates CCL3/CCR5-induced epithelial-to-mesenchymal transition via Erk1/2 and Akt signaling in cardiac myxoma
}

\author{
PING SHI ${ }^{1}, \mathrm{CHANGCUN} \mathrm{FANG}^{2}$ and XINYAN PANG ${ }^{2}$ \\ Departments of ${ }^{1}$ Emergency Surgery and ${ }^{2}$ Cardiosurgery, Qilu Hospital of Shandong University, Jinan, Shandong, P.R. China
}

Received May 8, 2015; Accepted June 9, 2015

DOI: $10.3892 /$ or.2015.4081

\begin{abstract}
In recent years, astrocyte elevated gene-1 (AEG-1) has been reported as a key mediator that is involved in the epithelial-to-mesenchymal transition (EMT) process. However, the mechanisms underlying CCL3/CCR5-AEG-1 pathway-mediated EMT in cardiac myxoma (CM) has not been well featured till now. We used immnohistochemistry and immunoblotting to assess the expression of CCR5 and AEG-1 in 30 cases of CM tissues and cells. Subsequently, cultured CM cells were treated with si-AEG-1 or si-CCR5 and then subjected to in vitro assays. We observed that CCR5 and AEG-1 proteins were highly expressed in CM tissues (73.3 and $76.7 \%$, respectively) and closely correlated with tumor size $(>5 \mathrm{~cm})$. Importantly, we validated the expression of AEG-1, p-Erk1/2, p-Akt, vimentin, N-cadherin and MMP2 increased in the CM cell with CCL3 treatment in a time- and concentration-dependent manner. When CM cells were treated with si-CCR5, the expression of AEG-1, p-Erk1/2, p-Akt, vimentin, N-cadherin and MMP2 was downregulated. In addition, when CM cells were treated with si-AEG-1, the expression of p-Erk1/2, p-Akt, vimentin, N-cadherin and MMP2 was also downregulated. Using the cell cycle and proliferation assay, the knockdown of AEG-1 inhibited the entry of G1 into S phase and the proliferation capacity of CM cells. In conclusion, AEG-1 mediates CCL3/CCR5induced EMT development via both Erk1/2 and Akt signaling pathway in CM patients, which indicates CCL3/CCR5-AEG1-EMT pathway could be suggested as a useful target to affect the progression of CM.
\end{abstract}

\section{Introduction}

Cardiac myxoma $(\mathrm{CM})$ is mostly sporadic in heart diseases $(1,2)$, and the clinical manifestation and long-term

Correspondence to: Dr Xinyan Pang, Department of Cardiosurgery, Qilu Hospital of Shandong University, No. 107 West Wenhua Road, Jinan, Shandong, P.R. China

E-mail: 13793182001@163.com

Key words: CCR5, AEG-1, epithelial-to-mesenchymal transition, cardiac myxoma complications include the recurrence, sudden death, heart failure and potential coronary embolisms $(3,4)$. Due to scarcity of studies indicating the molecular and signaling mechanisms in the CM development, it is very hard to realize the target therapy of CM. Generally surgical resection of CM mass is recommended as the only clinical regimen; however, this option has severe risk of death and can cause recurrences, infarction and stroke (5). Thus, it is necessary to throw light on the molecular mechanisms underlying CM progression, by which we can develop some new and useful targets agents.

It should be noted that astrocyte elevated gene-1 (AEG-1) can be produced in primary human fetal astrocytes (6). Recently, some studies proved that AEG-1 plays a crucial role in the pathogenesis, progression and invasion in different tumors (7-9). AEG-1 was also reported to induce the expression of E-cadherin and vimentin $(10,11)$, suggesting that AEG-1 may be involved in the regulation of epithelial-to-mesenchymal transition (EMT) (12). Moreover, CCR5, a G-protein-coupled receptor, activates cellular signaling cascades by binding to its ligand CCL3 $(13,14)$. CCL3/CCR5 axis promotes tumor development in various ways, including angiogenesis, modulation of extracellular matrix (15). However, the underlying mechanisms of CCL3/CCR5 in CM and the signaling pathways are not well known.

In the present study, we investigated and analyzed the biological roles of AEG-1 in CCL3/CCR5-induced EMT process using immunohistochemistry, immunoblotting, siRNA transfection and proliferation assay. Based on our results, we concluded that AEG-1 mediates CCL3/CCR5-induced EMT of $\mathrm{CM}$ via Erk1/2 instead of Akt signaling pathway, which indicates that CCL3/CCR5-AEG-1-Erk1/2-EMT pathway could be indicative of a useful target to benefit CM patients.

\section{Materials and methods}

Patients and tissues. Thirty left atrial myxomas were enrolled, including 14 male and 16 female patients (mean age, 53 \pm 5.8 ) who underwent surgery between August 2001 and August 2014. Gross assessment of CM tissues was carried out in order to measure the basic parameters such as tumor size. All pathological tissues were subjected to formalin fixation and paraffin-embedded. Prior to our medical research, the patients consent was obtained from the Institute Research Ethics Committee of Qilu Hospital of Shandong University. 
$C M$ cell culture. The culture of CM cells was conducted as previously mentioned in a published study (16). In vivo $\mathrm{CM}$ tissue was obtained from a 45-year-old patient who was diagnosed with sporadic CM, during surgical operation at Qilu Hospital. The patients gave informed consent before the operation, and the study design was approved and supported by the Qilu Hospital Medical Ethics Committee. The CM cells were harvested and separated by enzymatic digestion using collagenase and then were maintained in Dulbecco's modified Eagle's medium (DMEM). Prior to in vitro assays, the DMEM medium was changed with serum-free DMEM for another $24 \mathrm{~h}$, and then changed into fresh DMEM with the indicated reagents.

Reagents. Recombinant human CCL3 was purchased from Sigma (St. Louis, MO, USA). Antibodies were purchased from Santa Cruz Biotechnology (Santa Cruz, CA, USA). The specific antibodies included anti-p-Erk1/2, anti-t-Erk1/2, anti-p-Akt, anti-AEG-1, anti-N-cadherin, anti-MMP2 and anti- $\alpha$-tubulin antibody (Santa Cruz Biotechnology). All experiments were conducted in the absence of fetal bovine serum (FBS).

Immunohistochemistry. All sections were dewaxed in xylene and rehydrated in graded ethanol, followed by incubating in $3 \%$ hydrogen peroxide for $10 \mathrm{~min}$ to quench endogenous peroxides. Samples were heated in $0.01 \mathrm{~mol} / \mathrm{l}$ citrate buffer for $15 \mathrm{~min}$ at $100^{\circ} \mathrm{C}$, and then were placed at room temperature for $30 \mathrm{~min}$. After cooled, samples were blocked with $2 \%$ normal goat serum in phosphate-buffered saline (PBS) for 30 min to block antigenic epitopes, then incubated with primary antibody (1:100 dilution; Santa Cruz Biotechnology) at $4^{\circ} \mathrm{C}$ overnight. They were washed with PBS for 3 times, and then the samples were incubated with system-labeled HRP anti-mouse secondary antibody (Dako, Denmark) at room temperature for $20 \mathrm{~min}$. Next, the sections were incubated in DAB and counterstained in Mayer's hematoxylin, dehydrated in alcohol and xylene. The positive-stained slices (Santa Cruz Co.) were assessed from and PBS was used as negative control. Under the microscope, the positive areas appeared as brown yellow granules.

Evaluation of immunohistochemistry staining. The score of the immunohistochemistry staining was evaluated by one investigator who was blinded to the present study. The sections were scored based on the positive percentage and staining intensity. Sections were defined as positive if there were substantial amount of brown yellow granules in the plasma of the cells. The intensity of plasma staining was scored and graded as: 0 ( $0 \%$ cells); 1 ( $0-25 \%$ cells); 2 (25-50\% cells); and 3 (>50\% cells). Staining intensity was also evaluated semi-quantitatively as: 0 (none), 1 (mild), 2 (moderate), 3 (intense). The total score for each section was then evaluated by multiplying the intensity and positive percentage score, and was classified respectively into four levels: $0(-), 1-3(+), 4-6(++)$ and 7-9 (+++). The score was considered negative or low expression when the total was $<4$, and positive or high expression when it was $\geq 4$.

siRNA transfection. AEG-1 and CCR5 siRNA (a generous gift from Dr Wei Wang in Chinese Academy Science) transfection was performed as previously described. Cells were plated in 24-well plates. After $24 \mathrm{~h}$, the cells were transfected with control siRNA or with AEG-1/CCR5 siRNA using siRNA transfection reagent according to the manufacturer's instructions. The following siRNA sequences were targeted: AEG-1-siRNA positive sense, 5'-GGCAGGTATCTTTGTAA CTA-3' and antisense, 5'-GCTGACTGATTCTGGTTCAT-3'; CCR5 siRNA positive sense, 5'-GUUCAGAAACUACCUCU UAGUCUUCUUC-3' and antisense, 3'-UUCAAGUCUUUGA UGGAGAAUCAGAAGAAG-5'; control siRNA-positive sense, 5'-CAACCUUGCGGCCUUAGGGTT-3' and antisense, 5'-UUGGCCCAAUUUCCCGGGCTT-3'.

Immunoblotting. Total cell protein was extracted using a commercial kit, and the protein concentration was assessed by the BCA protein assay. Subsequently $30 \mu \mathrm{g}$ of denaturalized protein was separated by $10 \%$ SDS-polyacrylamide gel electrophoresis (SDS-PAGE), and then transferred into polyvinylidene fuoride membranes (Millipore). The protein was blocked with $5 \%$ skim milk in Tris-buffered saline containing $0.1 \%$ Tween-20 (TBST) for $2 \mathrm{~h}$ at room temperature. The protein was treated overnight with primary antibodies at $4^{\circ} \mathrm{C}$. After washed in TBST 3 times, the polyvinylidene fuoride membrane was incubated with horseradish peroxidase (HRP)-conjugated secondary antibody for another $2 \mathrm{~h}$. Finally, the protein bands were measured using the ECL detection system.

Cell cycle distribution analysis. After cells were seeded for $24 \mathrm{~h}$, the cells reached $\sim 75 \%$ confluence and were then treated with CCL3 at concentrations of $30 \mu \mathrm{M}$ for $24 \mathrm{~h}$. Following the treatment, cells were detached and fixed with $70 \%$ ethanol at $-20^{\circ} \mathrm{C}$ overnight. Subsequently, the cells were collected by centrifugation at $250 \mathrm{x}$ g for $5 \mathrm{~min}$. Then, the cells were washed with PBS and incubated with $25 \mu \mathrm{g} / \mathrm{ml}$ RNase A and $50 \mu \mathrm{g} / \mathrm{ml}$ PI for $30 \mathrm{~min}$ in the dark. A total number of $1 \times 10^{4}$ cells were subject to cell cycle analysis using a flow cytometry (BectonDickinson Immunocytometry Systems, San Jose, CA, USA).

Proliferation assays. The proliferation assays were performed as reported previously (16). Cells were seeded into plastic wells and grown for $48 \mathrm{~h}$ in DMEM with $10 \%$ FBS. After $24 \mathrm{~h}$, cells were treated with different IGF-1 treatment for $48 \mathrm{~h}$ with high serum conditions, and then exposed to DMEM containing $1 \mathrm{mCi}\left[{ }^{3} \mathrm{H}\right]$-thymidine for the next $24 \mathrm{~h}$. The cells were washed, obtained and counted in a liquid scintillation counter.

Statistical analysis. Data are expressed as the mean \pm standard error (SEM) of repeated assays. The correlation between CCR5 and AEG-1 was analyzed using Spearman's test. Significant differences between the two groups were assessed using $\chi^{2}$ analysis, Student's t-test and one-way ANOVA. In the present study, the $\mathrm{p}<0.05$ was considered to indicate a statistically significant result.

\section{Results}

Expression of CCR5 and AEG-1 in the CM tissue and cells. To identify the potential CCR5-AEG-1-EMT pathway in CM tissues, we introduced immunohistochemistry to investigate 


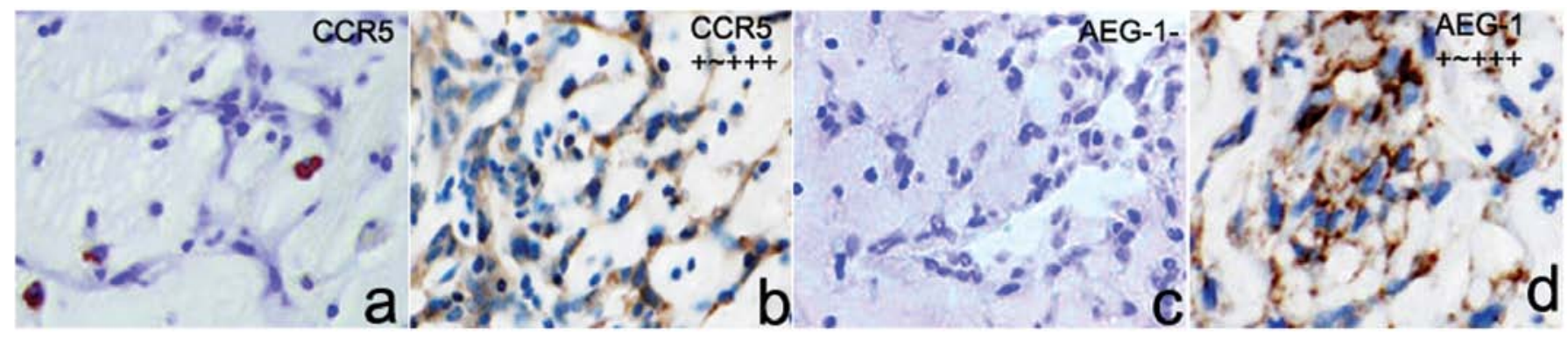

Figure 1. Expression of CCR5 and AEG-1 protein in CM tissues. Immunohistochemical staining was conducted in all 30 CM tissue sections using anti-CCR5 or anti-AEG-1 antibodies; at the same time, the representative image is shown. (a and b) CCR5 and (c and d) AEG-1 protein showed different expression intensities $(-,+\sim+++)$; positive immunostaining indicates the tumor cells of CM tissues. AEG-1, astrocyte elevated gene-1; CM, cardiac myxoma.

Table I. Correlation of CCR5, AEG-1 expression with clinicopathological features of cardiac myxoma.

\begin{tabular}{|c|c|c|c|c|c|c|c|}
\hline \multirow[b]{2}{*}{ Indicators } & \multirow[b]{2}{*}{ Case } & \multicolumn{2}{|c|}{ CCR5 } & \multirow[b]{2}{*}{ P-value } & \multicolumn{2}{|c|}{ AEG-1 } & \multirow[b]{2}{*}{ P-value } \\
\hline & & High & Low & & High & Low & \\
\hline \multicolumn{8}{|l|}{ Age (years) } \\
\hline$<60$ & 10 & 8 & 2 & 0.559 & 8 & 2 & 0.760 \\
\hline$\geq 60$ & 20 & 14 & 6 & & 15 & 5 & \\
\hline \multicolumn{8}{|l|}{ Gender } \\
\hline Male & 14 & 10 & 4 & 0.825 & 11 & 3 & 0.818 \\
\hline Female & 16 & 12 & 4 & & 12 & 4 & \\
\hline \multicolumn{8}{|l|}{ Location } \\
\hline Left atrium & 26 & 20 & 6 & 0.257 & 20 & 6 & 0.933 \\
\hline Right atrium & 4 & 2 & 2 & & 3 & 1 & \\
\hline \multicolumn{8}{|c|}{ Tumor size $(\mathrm{cm})$} \\
\hline$<5$ & 16 & 9 & 7 & 0.024 & 9 & 8 & 0.003 \\
\hline$\geq 5$ & 14 & 13 & 1 & & 14 & 0 & \\
\hline
\end{tabular}

AEG-1, astrocyte elevated gene-1.

the expression level of CCR5 and AEG-1 in 30 cases of $\mathrm{CM}$ tissues. As shown in Fig. 1, we found that 22 cases in all $30 \mathrm{CM}$ tissues highly expressed CCR5 protein, and the expression rate was $73.3 \%$. However, CCR5 protein was rarely stained in surrounding non-tumor samples. Similar with CCR5 expression model, 23 cases of CM tissues expressed AEG-1 protein and the expression ratio was $76.7 \%$. We also demonstrated that AEG-1 protein was not constitutively express in surrounding tissues. Under the microscope, CCR5 and AEG-1 protein was mainly located at the cell membrane and cytoplasm. Based on the Spearman's correlation analysis, high CCR5 expression was closely correlated to high AEG-1 expression in $\mathrm{CM}$ tissues $(\mathrm{r}=0.919, \mathrm{p}=0.001)$.

Correlations between CCR5, AEG-1 expression and clinicopathology. We analyzed the correlation between CCR5, AEG-1 expression and clinicopathology. As described in Table I, tumor size ( $>5 \mathrm{~cm}$ in diameter) was significantly associated with high-CCR5 expression $(\mathrm{p}=0.024)$, yet age and location had no significant association with the CCR5 expression. Likewise, tumor size ( $>5 \mathrm{~cm}$ in diameter) was also closely associated with high AEG-1 expression ( $\mathrm{p}=0.003)$, however, age, gender and tumor location had no discernible associations with the AEG-1 expression.

CCL3/CCR5 activates AEG-1 signaling and the EMT process in cultured CM cells in a time- and dose-dependent manner. To investigate the potential CCL3/CCR5 signaling pathway in the EMT process, we cultured CM cells with different CCL3 concentrations $(0,5,10,20,30$ and $40 \mathrm{ng} / \mathrm{ml})$, and then carried out immunoblotting to assess the expression status of the downstream signaling protein p-Erk1/2, p-Akt and AEG-1. As shown in Fig. 2a, immunoblotting results identified that the expression of p-Erk1/2, p-Akt and AEG-1 was highly upregulated in CCL3-induced CM cells in a dose-dependent manner. Besides, we also assessed the EMT biomarkers vimentin, $\mathrm{N}$-cadherin and MMP2. Our results showed that the protein expression of vimentin, $\mathrm{N}$-cadherin and MMP2 was aberrantly increased in CCL3 treatment, which was also in a dosedependent manner. In addition, we assessed the appropriate time when the downstream protein and EMT biomarkers in the cells can be activated. We found that CCL3 activates cells 

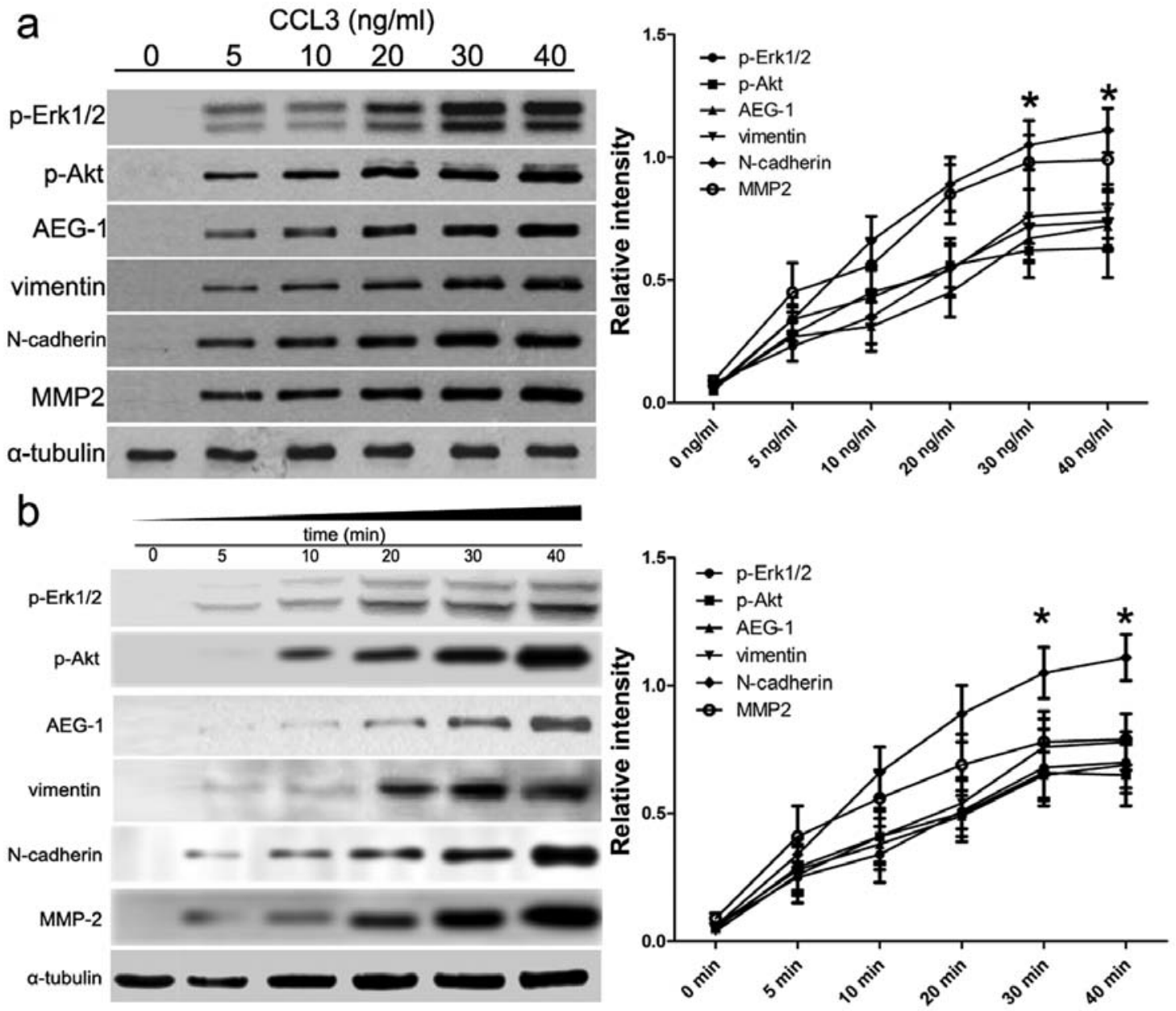

Figure 2. Effects of CCL3/CCR5 axis on AEG-1 signaling pathways and EMT. (a) Cells were maintained in serum-free medium for 30 min, and were then treated with human recombinant CCL3 at different concentrations. After 30 min treatment, cells lysates were subjected to immunoblotting. Subsequently, CCL3 signaling pathway-related molecules and EMT biomarkers were assessed. Protein expression level was calculated using ImageJ Pro software. $\alpha$-tubulin was used as a normalization control. (b) Cells were maintained in serum-free medium for $30 \mathrm{~min}$, and then treated with human recombinant CCL3 using $30 \mathrm{ng} / \mathrm{ml}$ of CCL3. Then, cells lysates were subjected to immunoblotting at different time points $(0.5,10,20,30$ and 40 min). Subsequently, CCL3 signaling pathway-related molecules and EMT biomarkers were assessed. Protein expression level was calculated using ImageJ Pro software. $\alpha$-tubulin was used as a normalization control. Each bar represents the mean \pm SEM of 3 independent experiments; " $p<0.001$, compared with si-control. AEG-1, astrocyte elevated gene-1; EMT, epithelial-to-mesenchymal transition.

time-dependently and 30 min treatment is better than the other time points. The treatment of 30 and 40 min showed no differences ( $p=0.356)$ (Fig. 2b). These results indicate that CCL3 activates AEG-1 signaling and the EMT process in CM cells, and the detailed pathways should be identified using gene silencing.

Knockdown of CCR5 abrogates CCL3-induced AEG-1 signaling and $E M T$. To further investigate the potential CCL3/CCR5 signaling pathway in the EMT process we cultured CM cells with CCR5 siRNA, then treated with CCL3 treatment $(30 \mathrm{ng} / \mathrm{ml})$, and then carried out immunoblotting to assess the expression status of the downstream signaling protein p-Erk1/2, p-Akt and AEG-1. As shown in Fig. 3, immunoblotting results identified that the expression of p-Erk1/2, p-Akt was obviously inhibited in CCR5 siRNA-induced CM cells. Besides, we also assessed the EMT biomarkers vimentin, $\mathrm{N}$-cadherin and MMP2. Our findings showed that the protein expression of vimentin, N-cadherin and MMP2 is obviously affected. These results indeed indicate that CCL3 activates AEG-1 signaling and the EMT process in cultured CM cells.

Knockdown of AEG-1 abrogates CCL3-induced EMT. Based on the above studies, we assumed that AEG-1 played a key role in regulating CCL3-induced EMT. To this end, we introduced AEG-1 siRNA to silence the mRNA transcription of AEG-1 gene (Fig. 4a), and then performed immunoblotting assay to assess downstream signaling molecules in the CCL3 pathway as well as EMT biomarkers as previously mentioned. As shown in Fig. 4b and c, our results showed the expression of p-Erk1/2, p-Akt, vimentin, N-cadherin and MMP2 increased in CM cells treated with control siRNA or CCL3 alone, which was consistent with our previous conclusion. By contrast, once we transfected AEG-1 siRNA into CM cells, the expression of p-Erk/2, p-Akt, vimentin, N-cadherin and MMP2 was obviously decreased. These results suggest that AEG-1 acts as a key mediator in CCL3-induced Erk1/2 and Akt signaling, and then regulates the progression of EMT. 
a

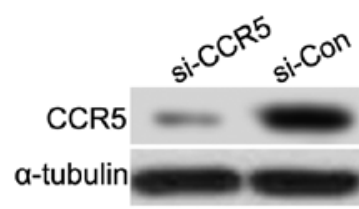

C

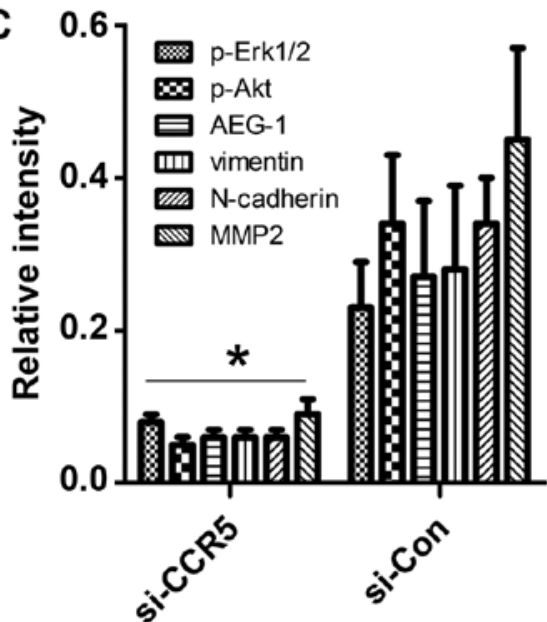

b

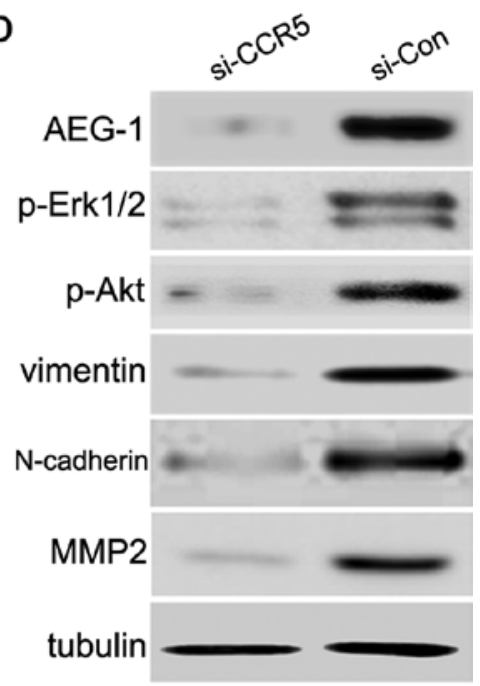

Figure 3. Effects of silencing of CCR5 on CCL3-induced signaling and EMT. CM cells were transfected with si-control or si-CCR5. At $48 \mathrm{~h}$ post-transfection, cells were treated with $30 \mathrm{ng} / \mathrm{ml}$ of CCL3. (a and b) Then cell lysates were subjected to immunoblotting. CM cells transfected with CCR5 siRNA obviously abrogated CCL3-induced signaling pathways and the activation of EMT biomarkers. Protein expression level was calculated using ImageJ Pro software (c). $\alpha$-tubulin was used as a normalization control. Each bar represents the mean \pm SEM of 3 independent experiments; " $p<0.001$, compared with si-control. EMT, epithelial-to-mesenchymal transition; CM, cardiac myxoma.
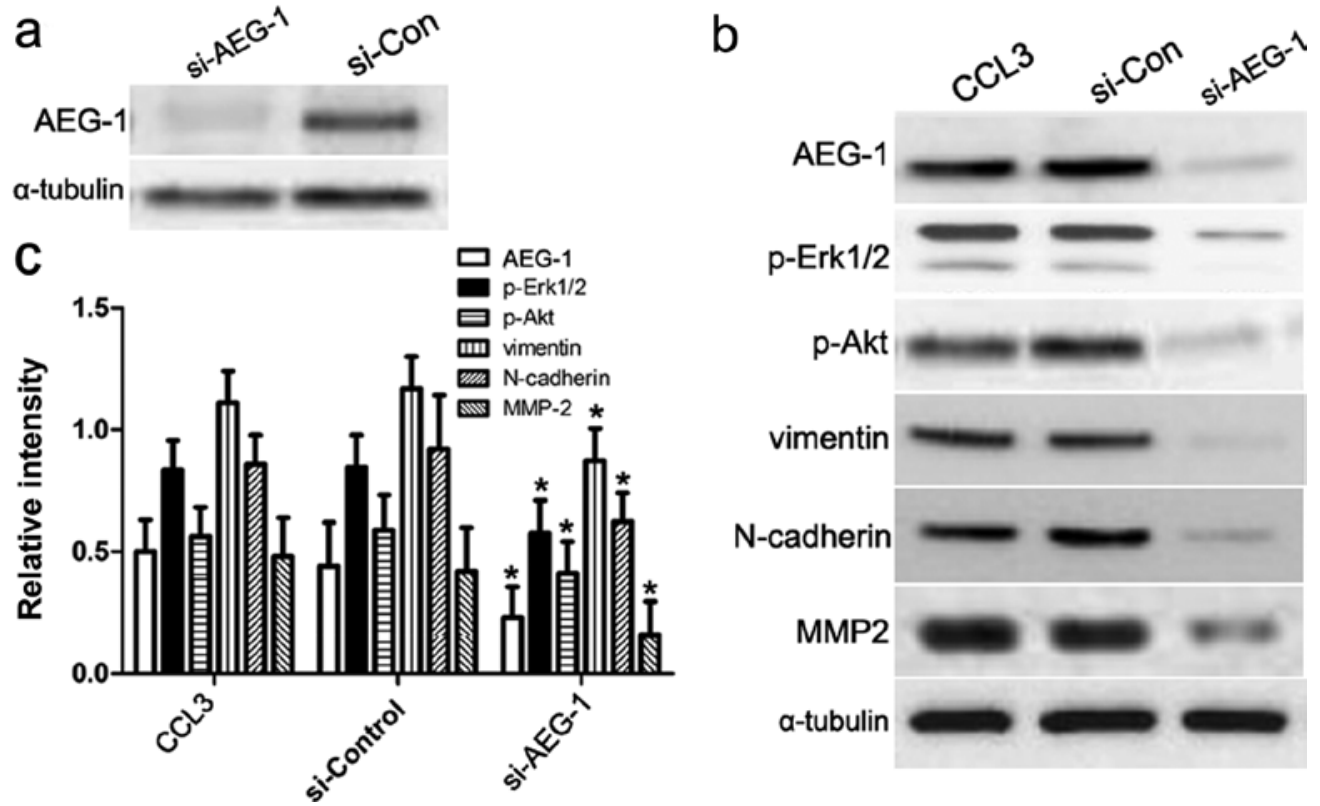

Figure 4. The effects of silencing AEG-1 on CCL3-induced signaling and EMT. CM cells were transfected with si-control or si-AEG-1. At $48 \mathrm{~h}$ post-transfection, cells were treated with $30 \mathrm{ng} / \mathrm{ml}$ of CCL3. (a and b) Then cells lysates were subjected to immunoblotting. CM cells transfected with AEG-1 siRNA obviously abrogated CCL3-induced signaling pathways and the activation of EMT biomarkers. Protein expression level was calculated using ImageJ Pro software (c). $\alpha$-tubulin was used as a normalization control. Each bar represents the mean \pm SEM of 3 independent experiments; ${ }^{*} \mathrm{p}<0.001$, compared with si-control. AEG-1, astrocyte elevated gene-1; CM, cardiac myxoma; EMT, epithelial-to-mesenchymal transition.

Silencing of either AEG-1 or CCR5 affects CCL3-induced CM cell cycle. Based on the above studies, we further assessed the effects of AEG-1 or CCR5 on cell cycle and the expression of cell cycle regulators, including CDK2, cyclin D1 in CM cells using flow cytometry and western blotting. Incubation of
CM cells with si-AEG-1 or si-CCR5 resulted in the inhibition of G1 phase entry into S phase (Fig. 5a). At the same time, the expression of CDK2 and cyclin D1 in CM cells was obviously suppressed in comparison with the control (Fig. 5b), indicating that AEG-1 is a key regulator in G1-to-S phase transition. 

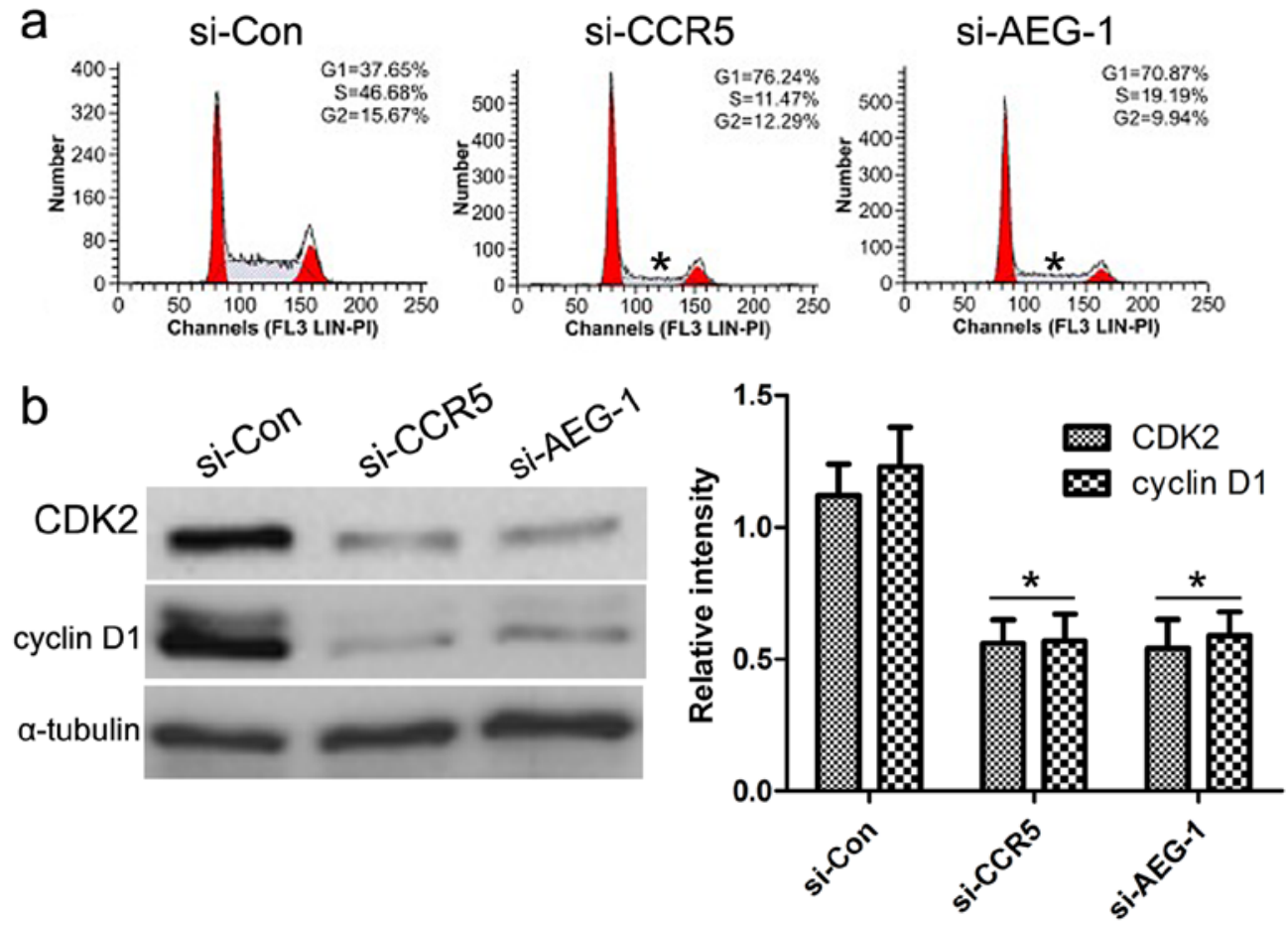

Figure 5. AEG-1 controls the cell cycle of CM cells. (a) Samples were taken following $12 \mathrm{~h}$ post-treatment and DNA content was assayed by flow cytometry and PI staining. The gates and percentages of cells in the S phase are indicated. (b) The expression of cyclin D1 and CDK2 in CM cells was subjected to western blotting. $\alpha$-tubulin acts as a control. Each bar represents the mean \pm SEM of 3 independent experiments; ${ }^{*} \mathrm{p}<0.001$, compared with control, one-way ANOVA or Student-Newman-Keuls. AEG-1, astrocyte elevated gene-1; CM, cardiac myxoma.

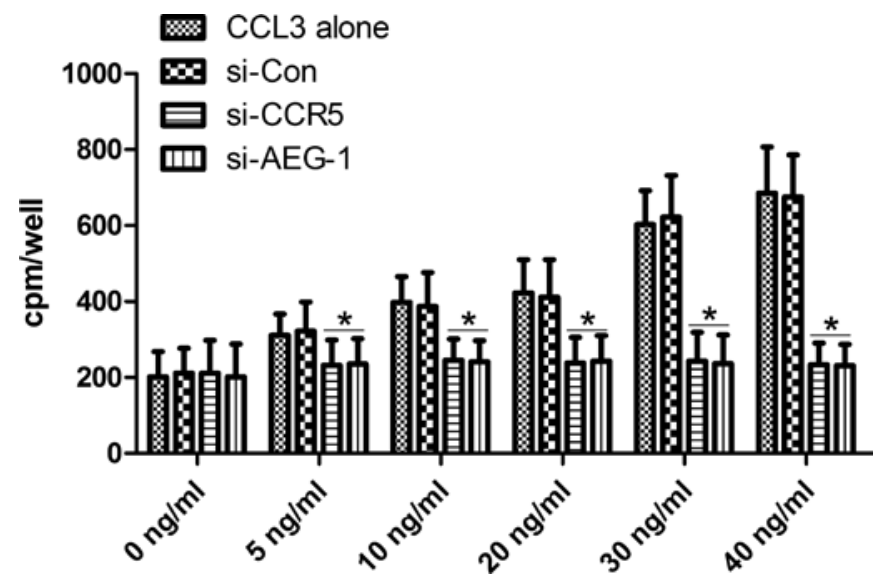

Figure 6. AEG-1 affected the proliferation of CM cells. After $24 \mathrm{~h}$ in serum-free medium, the cells were treated with CCL3, and then exposed to DMEM containing $1 \mathrm{mCi}\left[{ }^{3} \mathrm{H}\right]$-thymidine for another $24 \mathrm{~h}$. CCL3 caused a 2- to 5 -fold increase in $\left[{ }^{3} \mathrm{H}\right]$-thymidine incorporation concentration dependently. Knockdown of AEG-1 or CCR5 decreased DNA synthesis by $45-70 \%$ in a concentration-dependent manner and inhibited the proliferation of $\mathrm{CM}$ cells induced by CCL3. The measurements were performed in triplicate, and data are presented as mean $\pm \operatorname{SEM}(n=3)$. Significance vs. control, *p $<0.01$. AEG-1, astrocyte elevated gene-1; CM, cardiac myxoma.

Depletion of either AEG-1 or CCR5 affects CCL3-induced CM cell proliferation. To assess the impact of AEG-1 and CCR5 on proliferation behavior of $\mathrm{CM}$ cells, we investigated the potential roles of AEG-1 and CCR5 in CCL3-induced CM cell behavior using proliferation assay. In the present study, CCL3 treatment caused a 2- to 5 -fold increase in $\left[{ }^{3} \mathrm{H}\right]$-thymidine incorporation in a concentration-dependent manner $(\mathrm{p}<0.05)$. Unfortunately, this increase induced by CCL3 stimulation was abolished by knockdown of AEG-1 or CCR5. AEG-1 depletion decreased the constitutive proliferation of $\mathrm{CM}$ cells in a dose-dependent manner $(\mathrm{p}<0.05)$ (Fig. 6).

\section{Discussion}

In the published studies, CCL3/CCR5 axis has been reported to be involved in CM development. Moreover, AEG-1 is also reported to promote tumor progression, and involved in various growth factors, chemokines-induced signaling pathways $(17,18)$. In the present study, we tried to elucidate the role of AEG-1 in activation and regulation of CCL3-induced EMT pathway. Firstly, based on IHC results, high expression of CCR5 and AEG-1 protein was stained in major CM tissues. Pathological results proved that CCR5 and AEG-1 protein was significantly correlated with tumor size. In accordance with our results, the published studies identified that the CCR5 and AEG-1 were highly expressed in many other tumors, such as hepatocellular carcinoma (19) and also closely associated with the tumor size. All things considered, we assumed that CCR5-AEG-1 pathway was implicated in the progression of CM.

The EMT process was reported to play a key role in the tumorigenesis. The EMT process is characterized as the deregulation of cell-to-cell link systems and the consolidation of cellular migration and motility, making abnormally proliferating cells detached from the original epithelial tissues $(20,21)$. Recent studies showed that it is the high $\mathrm{N}$-cadherin expression that impairs E-cadherin regulated intercellular adhesion, and 
results in lack of epithelial barrier and deregulation of extracellular matrix (20). The multiple molecules involved in EMT exert a useful effect on cancer cell invasion, migration and dissemination. However the detailed molecular mechanisms of EMT have not been absolutely elucidated, thus we detailed the effects of CCR5-AEG-1 pathway on the EMT processes. Our findings showed that AEG-1, p-Erk1/2, p-Akt, vimentin, $\mathrm{N}$-cadherin and MMP2 were highly expressed once stimulated by CCL3. In contrast, when CM cells were transfected with AEG-1 siRNA, the expression of p-Erk/2, p-Akt, vimentin, $\mathrm{N}$-cadherin and MMP2 was obviously inhibited. These results indicate that CCL3 activates AEG-1 signaling and the EMT process via Erk1/2 and Akt pathways.

The mitogen-activated extracellular signal-regulated kinase Erk1/2 pathway is mostly featured as key signaling mediator in the signaling transduction of tumor biology. The Erk1/2 pathway can be activated by various factors, such as chemokines and inflammation mediators. More and more studies found the dysfunction of Erk1/2 signaling is the most common cause of cancer cell proliferation, which greatly promotes malignancy transformation (22). These kinases in EMT process offer some novel targets (23). As reported, the abnormal activation of AEG-1-Erk1/2 pathway in human sacral chondrosarcoma mediated cell proliferation and EMT progression (24). Moreover, the effects of PI3K/ Akt pathway on chemokine-induced EMT have been focused on $(25,26)$. However, few studies described the role of AEG-1 in Akt-induced EMT. In the present study, our results suggest that AEG-1 acts as a key mediator in CCL3-induced Erk1/2 pathway and the EMT process, as well as in Akt signaling.

Since functional analysis demonstrates the real effects of AEG-1, we conducted proliferation assays, and observed that CCL3 caused an obvious increase in $\left[{ }^{3} \mathrm{H}\right]$-thymidine incorporation in a concentration-dependent mode, and then $\left[{ }^{3} \mathrm{H}\right]$-thymidine incorporation was affected by knockdown of AEG-1. AEG-1 depletion decreased the constitutive proliferation of CM cells. These results indicate that AEG-1 interferes with CCL3-induced CM cell invasion and migration. In agreement with our studies, some studies on tumor biology also testified the significance of AEG-1 in the cell growth of tumor cells $(27,28)$.

In conclusion, our results suggest that AEG-1 mediates CCL3/CCR5-induced EMT of CM via both Akt and Erk1/2 signaling pathways in CM development, indicating that CCL3AEG-1-Erk1/2 and Akt-EMT pathways could be suggested as a prospective target to antagonize the $\mathrm{CM}$ progression, which can benefit $\mathrm{CM}$ patients in the clinical practice.

\section{Acknowledgements}

We greatly thank other members of our laboratory for valuable suggestions and writing.

\section{References}

1. Lee HS, Kim HK, Park EA, Kim KH, Kim YJ and Sohn DW: Left atrial intramural hematoma after removal of atrial myxoma: Cardiac magnetic resonance in the differential diagnosis of intracardiac mass. J Cardiovasc Ultrasound 22: 205-208, 2014.

2. Watanabe H, Nara I, Yamaura G, Iino K, Iino T, Shimbo M, Seki $\mathrm{K}$ and Ito $\mathrm{H}$ : Blood balloon induced by an atrial myxoma in the heart. Circulation 130: 2351-2353, 2014.
3. Anpalakhan S, Ramasamy D and Fan KS: An unusual presentation of atrial myxoma. Singapore Med J 55: e156-e158, 2014.

4. Choi K, Jung D, Hong SW, Jeon Y and Kim SO: Extra cardiac tumor misdiagnosed as a left atrial myxoma. Korean $\mathrm{J}$ Anesthesiol 67 (Suppl): S67-S68, 2014.

5. Di Vito A, Mignogna $C$ and Donato G: The mysterious pathways of cardiac myxomas: A review of histogenesis, pathogenesis and pathology. Histopathology 66: 321-332, 2015.

6. Su ZZ, Kang DC, Chen Y, Pekarskaya O, Chao W, Volsky DJ and Fisher PB: Identification and cloning of human astrocyte genes displaying elevated expression after infection with HIV-1 or exposure to HIV-1 envelope glycoprotein by rapid subtraction hybridization, RaSH. Oncogene 21: 3592-3602, 2002.

7. Yoo BK, Emdad L, Su ZZ, Villanueva A, Chiang DY, Mukhopadhyay ND, Mills AS, Waxman S, Fisher RA, Llovet JM, et al: Astrocyte elevated gene-1 regulates hepatocellular carcinoma development and progression. J Clin Invest 119: 465-477, 2009.

8. Lee SG, Jeon HY, Su ZZ, Richards JE, Vozhilla N, Sarkar D, Van Maerken T and Fisher PB: Astrocyte elevated gene-1 contributes to the pathogenesis of neuroblastoma. Oncogene 28: 2476-2484, 2009.

9. Hu G, Chong RA, Yang Q, Wei Y, Blanco MA, Li F, Reiss M, $\mathrm{Au}$ JL, Haffty BG and Kang Y: $M T D H$ activation by $8 \mathrm{q} 22$ genomic gain promotes chemoresistance and metastasis of poorprognosis breast cancer. Cancer Cell 15: 9-20, 2009.

10. Liu K, Guo L, Miao L, Bao W, Yang J, Li X, Xi T and Zhao W: Ursolic acid inhibits epithelial-mesenchymal transition by suppressing the expression of astrocyte-elevated gene-1 in human nonsmall cell lung cancer A549 cells. Anticancer Drugs 24: 494-503, 2013.

11. Li J, Zhang N, Song LB, Liao WT, Jiang LL, Gong LY, Wu J, Yuan J, Zhang HZ, Zeng MS, et al: Astrocyte elevated gene-1 is a novel prognostic marker for breast cancer progression and overall patient survival. Clin Cancer Res 14: 3319-3326, 2008.

12. Weber CE, Li NY, Wai PY and Kuo PC: Epithelial-mesenchymal transition, TGF- $\beta$, and osteopontin in wound healing and tissue remodeling after injury. J Burn Care Res 33: 311-318, 2012.

13. Soria $G$ and Ben-Baruch A: The inflammatory chemokines CCL2 and CCL5 in breast cancer. Cancer Lett 267: 271-285, 2008.

14. Mencarelli A, Graziosi L, Renga B, Cipriani S, D'Amore C, Francisci D, Bruno A, Baldelli F, Donini A and Fiorucci S: CCR5 antagonism by maraviroc reduces the potential for gastric cancer cell dissemination. Transl Oncol 6: 784-793, 2013.

15. Sasaki S, Baba T, Shinagawa K, Matsushima K and Mukaida N: Crucial involvement of the CCL3-CCR5 axis-mediated fibroblast accumulation in colitis-associated carcinogenesis in mice. Int J Cancer 135: 1297-1306, 2014.

16. Sakamoto H, Sakamaki T, Kanda T, Tsuchiya Y, Sato M, Sato H, Oyama Y, Sawada Y, Tamura J, Nagai R, et al: Vascular endothelial growth factor is an autocrine growth factor for cardiac myxoma cells. Circ J 68: 488-493, 2004.

17. He W, He S, Wang Z, Shen H, Fang W, Zhang Y, Qian W, Lin M, Yuan J, Wang J, et al: Astrocyte elevated gene-1 (AEG-1) induces epithelial-mesenchymal transition in lung cancer through activating Wnt/ $\beta$-catenin signaling. BMC Cancer 15: 107, 2015.

18. Zheng J, Li C, Wu X, Liu M, Sun X, Yang Y, Hao M, Sheng S, Sun Y, Zhang H, et al: Huaier polysaccharides suppresses hepatocarcinoma MHCC97-H cell metastasis via inactivation of EMT and AEG-1 pathway. Int J Biol Macromol 64: 106-110, 2014.

19. Khorramdelazad H, Mortazavi Y, Momeni M, Arababadi MK, Khandany BK, Moogooei M and Hassanshahi G: Lack of correlation between the CCR5- $\Delta 32$ mutation and acute myeloid leukemia in Iranian patients. Indian J Hematol Blood Transfus 31: 29-31, 2015.

20. Shi Y, Wu H, Zhang M, Ding L, Meng F and Fan X: Expression of the epithelial-mesenchymal transition-related proteins and their clinical significance in lung adenocarcinoma. Diagn Pathol 8: 89, 2013.

21. Shih JY and Yang PC: The EMT regulator slug and lung carcinogenesis. Carcinogenesis 32: 1299-1304, 2011.

22. Chowdhury I, Thompson WE and Thomas K: Prohibitins role in cellular survival through Ras-Raf-MEK-ERK pathway. J Cell Physiol 229: 998-1004, 2014.

23. Neuzillet C, Tijeras-Raballand A, de Mestier L, Cros J, Faivre S and Raymond E: MEK in cancer and cancer therapy. Pharmacol Ther 141: 160-171, 2014. 
24. Wang F, Ke ZF, Wang R, Wang YF, Huang LL and Wang LT: Astrocyte elevated gene-1 (AEG-1) promotes osteosarcoma cell invasion through the JNK/c-Jun/MMP-2 pathway. Biochem Biophys Res Commun 452: 933-939, 2014.

25. Zhou SL, Zhou ZJ, Hu ZQ, Li X, Huang XW, Wang Z, Fan J, Dai $Z$ and Zhou J: CXCR2/CXCL5 axis contributes to epithelial-mesenchymal transition of HCC cells through activating PI3K/Akt/GSK-3 $\beta /$ Snail signaling. Cancer Lett 358: 124-135, 2015.

26. Bhat FA, Sharmila G, Balakrishnan S, Arunkumar R, Elumalai P Suganya S, Raja Singh P, Srinivasan N and Arunakaran J: Quercetin reverses EGF-induced epithelial to mesenchymal transition and invasiveness in prostate cancer (PC-3) cell line via EGFR/PI3K/Akt pathway. J Nutr Biochem 25: 1132-1139, 2014.
27. Yao C, Lv S, Han M, Zhang J, Zhang Y, Zhang L, Yi R, Zhuang D and Wu J: The association of Crk-like adapter protein with poor prognosis in glioma patients. Tumour Biol 35: 5695-5700, 2014.

28. Srivastava J, Robertson CL, Rajasekaran D, Gredler R, Siddiq A, Emdad L, Mukhopadhyay ND, Ghosh S, Hylemon PB, Gil G, et al: AEG-1 regulates retinoid $\mathrm{X}$ receptor and inhibits retinoid signaling. Cancer Res 74: 4364-4377, 2014. 